\title{
Les peacelines de Belfast, entre maintien de l'ordre et gestion urbaine
}

The Belfast peacelines, between law enforcement and political negotiation

Florine Ballif

\section{(2) OpenEdition}

12 Journals

\section{Édition électronique}

URL : http://journals.openedition.org/conflits/17533

DOI : $10.4000 /$ conflits. 17533

ISSN : $1777-5345$

Éditeur :

CCLS - Centre d'études sur les conflits lilberté et sécurité, L'Harmattan

\section{Édition imprimée}

Date de publication : 30 mars 2009

Pagination : 73-83

ISBN : 978-2-296-07748-5

ISSN : 1157-996X

Référence électronique

Florine Ballif, «Les peacelines de Belfast, entre maintien de l'ordre et gestion urbaine », Cultures \&

Conflits [En ligne], 73 | printemps 2009, mis en ligne le 30 mars 2010, consulté le 30 mars 2021. URL:

http://journals.openedition.org/conflits/17533; DOI : https://doi.org/10.4000/conflits. 17533 


\section{Les peacelines de Belfast, entre maintien de l'ordre et gestion urbaine}

\section{Florine BALLIF}

Florine Ballif est diplômée de l'Institut d'études politiques de Strasbourg et docteur en urbanisme de l'université Paris-XII. Elle est chercheur associée à Habiter (EA 2076), université de Reims, où elle travaille sur les processus de fermeture des espacesurbains. Contact : ballif.f@neuf.fr

$\mathrm{P}$ resque vingt ans après la chute du mur de Berlin, la recrudescence de la fermeture des territoires et la prolifération des murs est un trait caractéristique du monde contemporain ${ }^{1}$. De nouveaux systèmes de fermeture et de contrôle des frontières sont édifiés en de nombreux points de la planète : sur la frontière Etats-Unis - Mexique, la barrière de sécurité entre Israël et Palestine à proximité de la « ligne verte ", à la périphérie des presidios espagnols de Ceuta et Melilla au Maroc, pour les plus connus. Les murs séparant des frontières territoriales coupent parfois des villes en deux, comme c'était le cas à Berlin et désormais à Chypre. Dans les espaces urbains ordinaires, les barrières aux formes variées se multiplient ${ }^{2}$. Les gated communities, quartiers fermés, transforment et cloisonnent le tissu urbain des grandes métropoles du Nord comme du Sud ${ }^{3}$.

Au-delà de l'apparence unitaire d'un phénomène de fermeture, la multiplicité des formes renvoie aux différents contextes d'apparition de ces artefacts qui permettent de cloisonner. Il peut s'agir de murs au sens strict (béton, briques), mais aussi de grillages (éventuellement électrifiés), renforcés par d'autres dispositifs, tels que la sirga, enchevêtrement de barres d'acier entre les clôtures, qui rend dange-

1. Novosseloff A., Neisse F., Des murs entre les hommes, Paris, La Documentation française, 2007.

2. Marcuse P., Walls of Fear and Walls of Support, Princeton Architectural Press, 1997.

3. Voir entre autres : Billard G., Chevalier J., Madoré F., Ville fermée, ville surveillée. La sécurisation des espaces résidentiels en France et en Amérique du Nord, Presses universitaires de Rennes, 2005 ; Le Goix R., « Les gated communities aux Etats-Unis et en France : une innovation dans le développement périurbain ", Hérodote, n¹22, 2006, pp. 107-137 ; Glasze G., Webster C., Frantz K. (eds), Private Cities: Global and Local Perspectives, Londres, Routledge, 2005.

4. Novosseloff A., Neisse F., op. cit., pp. 151-152. 
reuse la chute et impossible le déplacement dans ces interstices autour de l'enclave de Melilla ${ }^{4}$. D'éventuelles zones minées ou piégées - plus spécifiques aux frontières - sont installées et ces éléments en profondeur sont juxtaposés à des fossés et des routes de patrouilles. Aux points de franchissement, ces murs se caractérisent par la présence de checkpoints dotés de chevaux de frise, barrières et autres installations défensives. Sont parfois ajoutées des techniques moins visibles telles que les détecteurs infrarouges, les rayons, les ondes ou les modes de contrôles biométriques ${ }^{5}$. Dans certains cas, ces dispositifs se traduisent par «un allégement de la marque qui délimite et une intensification de l'action qui repousse ${ }^{6}$ ».

La multiplication de dispositifs apparents, de " murs », qui cloisonnent un espace pose la question de leur efficacité et de leur raison d'être. Au-delà du contrôle du territoire, les systèmes s'appuient sur toute une série de dispositifs qui ont pour but et pour effet de contrôler et restreindre le mouvement des individus. Les murs ne sont qu'un élément, le plus visible, mais de loin le plus central. Toutefois, le mur joue sans doute un rôle symbolique d'affirmation du contrôle. Il procède donc aussi bien de la prévention que d'une appropriation (ré)affirmée. De ce point de vue, il apparaît comme un dispositif logique dans la «société $d u$ risque 7 ». Pour Ulrich Beck, l'universalisation du risque et sa perception ont profondément modifié les représentations des acteurs politiques. L'attitude face au risque-comment le répartir, le gérer, l'éviter - devient un des déterminants fondamentaux des processus politiques et sociaux contemporains. Le risque créé par les guerres contemporaines « asymétriques » qui transcendent les frontières et les territoires signifie, pour Giorgio Agamben, que la "sécurité s'impose comme la principale activité de l'Etat » et comme «critère de légitimité politique » 8.

En Irlande du Nord, le phénomène est déjà ancien, puisque les premiers murs de sécurité datent de la fin des années 1960, et concerne principalement Belfast, la capitale, ainsi que les principales villes. Les murs ont été mis en place par l'armée britannique dans ses opérations de maintien de l'ordre. Ils sont un exemple assez atypique parce que les murs ne se superposent pas à des frontières internationales et qu'ils ne résultent pas non plus d'une ségrégation imposée brutalement. Mais le temps relativement long - cinquante ans cette année - permet de suivre leurs transformations et leur évolution, et, en cela, permet de contribuer à comprendre ce phénomène de fermeture qui semble se généraliser après le 11 septembre 2001.

5. Ceyhan A., «Technologie et sécurité : une gouvernance libérale dans un contexte d'incertitude », Cultures $\mathcal{E}$ Conflits, $\mathrm{n}^{\circ} 64$, hiver 2006, pp. 11-32.

6. Razac O., Histoire politique du barbelé : la prairie, la tranchée, le camp, Paris, La Fabrique, 2000, p. 86.

7 . Beck U., La Société du risque. Sur la voie d'une autre modernité, Paris, Aubier, 2001.

8. Agamben G., "Security and Terror", Theory and Event, vol.5, n4, 2002, p. 1, cité dans : Graham S. (ed), Cities, War and Terrorism: Towards an Urban Geopolitics, Oxford, Blackwell, 2004, p. 4. 
Ils apparaissent comme une solution à court terme d'une situation où des violences civiles échappent au contrôle de l'Etat, mais cette solution entérine la situation de conflit, du moins la problématique de conflit territorial. Les murs ne sont pas démolis malgré la signature des accords de paix en 1998, et au contraire, de nouveaux sont construits.

Cet article se propose d'analyser les processus politiques qui ont abouti à la création de murs et à leur maintien, et les nouvelles significations que ce maintien apporte. Ces murs, au départ issus de la stratégie militaire et répondant à des objectifs de maintien de l'ordre suite à des émeutes violentes, ont constitué une tentative des acteurs politiques locaux de gestion locale et localisée du conflit intercommunautaire, passant d'une gestion militaire à une gestion civile, policière puis « urbaine » 9 .

\section{Le conflit ethno-national}

La «frontière irlandaise » a été établie en 1920 en Irlande du Nord, née de l'opposition entre les partisans de l'indépendance et ceux du maintien de l'île sous tutelle britannique. Elle a eu pour conséquence de «mettre en cage » les revendications territoriales des deux groupes ethno-nationaux, désormais circonscrites à l'Irlande du Nord ${ }^{10}$. Alors qu'elle a empêché le conflit de s'étendre, la partition de l'Irlande a institutionnalisé un profond antagonisme entre deux groupes inégaux, deux communautés aux aspirations politiques opposées et aux identités sociales, religieuses, économiques différentes : nationalistes irlandais catholiques et unionistes britanniques protestants ${ }^{11}$. La démocratie représentative a reproduit les lignes de division autour desquelles l'Irlande du Nord a été créée et s'est construite sur la question de la frontière. Le régime politique s'appuyait sur le contrôle ethno-territorial et était renforcé par un « apartheid volontaire $»$ des « deux communautés $» 12$.

Le conflit s'est transformé en guerre civile larvée à la fin des années 1960 - «les Troubles », touchant principalement les villes. La ville est souvent le microcosme de tensions et de fragmentations plus larges ${ }^{13}$. Boal a décrit Belfast comme la «miniature urbaine du conflit national 14 ». Une partie

9. Cet article s'appuie sur un travail de terrain effectué entre 1998 et 2002 dans le cadre d'une recherche doctorale. L'essentiel de l'analyse est située dans ces périodes.

10. O'Dowd L., Mc Call C., "Escaping the Cage of Ethno-National Conflict in Northern Ireland? Importance of Transnational Networks", Ethnopolitics, vol.7, n¹, 2008, p. 85.

11. Ils seront désignés dans l'article comme catholiques et protestants, ce qui est la terminologie la plus courante utilisée pour s'auto-désigner en Irlande du Nord.

12. O’Dowd L., Mc Call C., op. cit., p. 86.

13. Soja E., Postmetropolis, Critical Studies of Cities and Regions, Oxford, Blackwell, 2000.

14 . Boal F.W., Murray R.C., "Belfast: The Urban Encapsulation of a National Conflict", in Clarke S.C., Obler J.L. (eds), Urban Ethnic Conflict, a Comparative Perspective, Chapel Hill, Institute for Research in Social Science, University of North Caroline, Comparative urban studies monograph series $n^{\circ} 3,1977$, p. 77. 
importante du conflit s'est déroulée dans les rues de la capitale. Poole montre que Belfast concentre $54,5 \%$ des décès et $73,1 \%$ des incidents urbains liés aux Troubles pour la période 1969-1993, alors qu'elle ne représente que le tiers de la population ${ }^{15}$. La violence intercommunautaire est un facteur déterminant dans la répartition spatiale des communautés, et Belfast s'est développé en espaces appropriés de manière différentielle par les deux groupes. Les affrontements entre les communautés catholiques et protestantes prennent la forme d'émeutes, de combats de rue et d'une guérilla urbaine entre les milices paramilitaires et les forces de l'ordre. Ainsi Belfast est une ville polarisée selon Boal, car les divisions sociales, économiques et ethniques sont pensées comme des divisions nationales. D’après Bollens, ces identités ethno-nationales sont renforcées car elle peuvent s'inscrire dans l'espace urbain ${ }^{16}$. Cela correspond à la définition des nouvelles guerres urbaines que donne Arjun Appadurai, conflits "qui lient les politiques supranationales au micro politiques de la rue et des quartiers 17 ».

\section{Les premières peacelines, artefact du maintien de l'ordre}

En réponse aux émeutes de grande ampleur de l'été 1969 à Belfast, l'armée britannique adopte une stratégie de quadrillage militaire provisoire. Elle installe ce que le général Freeland, commandant des forces armées, appelle une peaceline - «mur de paix »-sur le théâtre principal des affrontements entre les quartiers ouvriers des Falls et du Shankill, bastions emblématiques des deux communautés, en remplacement des barricades érigées par la population. Le terme sera consacré. Mais il ne s'agit alors que de barbelés et de chevaux de frise, déterminant des zones patrouillées et surveillées par l'armée. Les vastes mouvements de population provoqués par la violence et la peur ${ }^{18}$ ont clarifié les limites des quartiers. Sur les lieux d'affrontement, des rues entières de maisons en bandes sont laissées en ruines, faisant place à des terrains en friche après démolition, sur lesquels seront édifiées au fur et à mesure des peacelines formalisées et structurées, en béton et en briques.

Les peacelines sont le résultat de décisions non planifiées, dans le cadre d'opérations de maintien de l'ordre de l'armée, puis à la discrétion de la police après le milieu des années 1970. La chronologie de leur construction échappe donc aux

15. Poole M., “The Spatial Distribution of Political Violence in Northern Ireland: an Update to 1993”, in O'Day A. (ed), Terrorism's Laboratory : The Case of Northern Ireland, Aldershot, Dartmouth Publishing Company, 1995, p. 31.

16. Bollens S., Urban Peace Building in Divided Socities: Belfast and Johannesburg, Colorado, Westview Press, 1999, p. 35.

17. Appadurai A., Fear of Small Numbers: An Essay on the Geography of Anger, Duke University Press, pp. 152-153, cité dans Graham S., op. cit., p. 7.

18. 60000 personnes se sont déplacées, cherchant refuge dans leurs quartiers confessionnels, ce qui représente 12 \% de la population. Voir Darby J., Morris G., Intimidation in Housing, Belfast, NI Community Relations Commission, 1994. 
archives et semble bien difficile à retracer. Les premières peacelines datent de 1969-1970. Bien que le processus soit continu, on peut identifier une deuxième vague de construction au milieu des années 1980, une autre à partir de la seconde moitié des années 1990 et, enfin, au début des années 2000. Leur nombre augmente avec le temps, mais n'est pas aisé à déterminer. En effet, les manières de recenser les peacelines varient. Ce que certains décrivent comme deux barrières sont pour d'autres une seule barrière continue, malgré les éventuels sectionnements. Cette incertitude est visible sur les documents officiels des administrations concernées. Même les services centraux du ministère de l'Intérieur (NIO) et les officiers de renseignements généraux avancent des chiffres contradictoires ${ }^{19}$.

L'association Belfast Interface Project met à disposition du public les chiffres du NIO et cartographie les peacelines sur son site Internet. Selon elle, le nombre de peacelines s'élève à 15 en 1994, date des cessez-le-feu de l'IRA et des milices paramilitaires protestantes, et à 17 en $1998^{20}$, date des accords de paix. Il y aurait 41 murs en $2008{ }^{21}$. D'après nos recherches, en nous appuyant sur un document de recension de 15 dispositifs 22 et sur nos observations de terrain, nous estimons que ces structures totalisent environ $7 \mathrm{~km}$ en 1998. Michel Foucher annonce que ces dispositifs se développent sur un total de $20 \mathrm{~km}$ en 200723.

Le cloisonnement urbain a des contours incertains. Les peacelines, situées dans les quartiers péricentraux de Belfast sur les limites entre enclaves catholiques et protestantes ne sont toujours pas des structures réellement closes. En effet, la fermeture n'est que partielle dans la mesure où un système de portails pour les piétons et les automobiles autorise un passage discontinu dans le temps et dans l'espace.

\section{Une stratégie de maintien des territoires confessionnels ?}

La construction des murs participe au processus de régulation de la différence socio-politique, ce qui expliquerait à la fois la volonté de ne pas les détruire de la part de la population et leur banalisation par les organismes à qui

19. En 1994, un rapport non publié réalisé par un groupe de travail du ministère de l’Intérieur nord-irlandais, le Northern Ireland Office (NIO) identifie quinze lieux où un mur ou une barrière sépare les deux communautés. En 1999, le même NIO fournit une liste de trente et un dispositifs à l'association Belfast Interface Project. Mais, en 2000, les agents de renseignement généraux, comptent vingt-sept murs ou barrières dans une note d'information au gouvernement.

20. Belfast Interface Project, List of NIO-built peace lines, 1998.

21 . Belfast Interface Project, Interface map (Carte interactive), http://www.belfastinterfaceproject.org/interfacemap.asp, consulté le 5 janvier 2008.

22. Environmental Design Consultants, Belfast Peacelines Study, Belfast, 1991. Il s'agit d'une étude commanditée par le Northern Ireland Housing Executive, NIHE (Agence nord-irlandaise pour le logement social) à un bureau d'études privé, EDC, et consiste en une analyse formelle des quinze peacelines recensées.

23 . Foucher M., L'Obsession des frontières, Paris, Perrin, 2007, p. 126. 
est confiée leur gestion. L'affrontement politique entre les deux communautés est guidé par un jeu à somme nulle dans le contrôle territorial et, par conséquent, la capacité à occuper ou à revendiquer un territoire est centrale ${ }^{24}$. En effet, l'un des principaux points de contestation et de crispation entre les communautés sur le plan urbain est le maintien des territoires confessionnels, alors que les deux communautés ont des dynamiques démographiques contradictoires. En fixant des limites aux territoires catholiques et protestants, très morcelés, les murs rendent le découpage manifeste et contribuent à ralentir les effets des pressions démographiques sur la carte confessionnelle de Belfast. Les murs sont situés pour la plupart sur des parcelles appartenant à l'organisme de construction et de gestion du logement social d'Irlande du Nord, le Nothern Ireland Housing Executive (NIHE). Le parc social représente environ un tiers du parc total de logement à Belfast, et plus de $40 \%$ de l'inner city. Le gestionnaire du logement social est particulièrement concerné par cette problématique.

Les deux communautés ont une dynamique démographique différenciée. La population protestante est plus vieillissante. En termes de mobilité intra-urbaine, les protestants s'installent en périphérie tandis que les catholiques restent dans l'inner city. La «fuite des protestants » vers la banlieue est liée à la perception selon laquelle ces espaces sont sûrs. Ainsi, le quartier protestant emblématique de Belfast, le Shankill, passe de 76000 habitants dans les années 1960 à 26000 dans les années 1990. La dépopulation de Belfast - de 416679 habitants en 1971 à 305763 en 1981 - est due en grande partie à un délaissement du centre pour les villes satellites ${ }^{25}$, dont la population totale augmente de 47118 habitants dans la même période. Cependant, la baisse de population s'explique autant par les politiques de rénovation urbaine que par le conflit et le déclin économique ${ }^{26}$. Aujourd'hui, les protestants, s'ils le peuvent, quittent le parc social situé dans les espaces centraux en déclin pour le logement privé ou demandent leur transfert dans des lotissements en périphérie. Le territoire catholique est plus restreint, limité à l'ouest et au nord de Belfast. Les zones protestantes du centre de Belfast se vident, alors qu'une forte pression s'exerce sur les secteurs catholiques. Les catholiques ocupent un territoire restreint déjà très densifié et qui ne peut être étendu. Dans un contexte de lutte pour les ressources qui structure les relations entre les deux groupes, chaque communauté souhaite maintenir et solidifier son territoire. La demande de logement est sous-tendue par une logique ethno-territoriale dans laquelle le parc social, subventionné par des fonds publics, est considéré comme l'espace réservé d'une communauté. Les catholiques veulent des nouveaux logements pour satisfaire les besoins de leur population croissante et accusent le NIHE de ne pas s'étendre dans les zones protestantes, sous-utilisées ${ }^{27}$. En revanche, les protestants veulent la stabilité et prônent la construction de nou-

24. O’Dowd L., Mc Call C., op. cit., p. 86.

25. Antrim, Newtownards, Ballymena, Carrickfergus, Craigavon, Lisburn et Newtownabbey.

26. Voir Wiener R., The Rape and Plunder of the Shankill in Belfast: People and Planning, Belfast, Notheams, 1976. 
veaux logements pour revitaliser les communautés. Le NIHE est parfois accusé d'engager un processus de «déprotestantisation» de la ville. Ses intentions seraient de laisser se dégrader les quartiers protestants pour que les locataires demandent leur transfert en périphérie. Cela entraîne une cristallisation des conflits autour des enclaves protestantes situées dans Belfast-nord et Belfast-ouest. Le NIHE est parfois critiqué pour ses intentions de faire du social engineering, c'est à dire de créer des espaces confessionnels plus facilement gérables. Mais, au contraire, la force du sentiment identitaire local est telle que les pouvoirs publics ont accepté, certes officieusement, qu'il ne soit pas possible de déplacer les peacelines pour construire des logements ou réduire l'éventualité des désordres ${ }^{28}$. Les gestionnaires du NIHE sont conscients que vouloir modifier la structure territoriale de Belfast comporte de trop grands risques.

L'expérience malheureuse de Manor Street en 1985 est citée par les cadres $\mathrm{du}$ NIHE comme un contre-exemple à ne jamais reproduire ${ }^{29}$. De nouveaux logements sociaux avaient été construits du côté protestant de cette interface de Belfast-nord. Quinze logements vacants, faute de demande locale, avaient été attribués à des catholiques, très nombreux sur la liste d'attente du lotissement catholique attenant. Le NIHE reculait ainsi la limite territoriale d'une rangée de maisons, les jardins à l'arrière de ces nouveaux logements attribués à des catholiques marquant la frontière. En réaction, les paramilitaires protestants ont empêché l'installation des familles catholiques. Les émeutes qui ont suivi ont entraîné le pillage, puis la démolition de ces logements ${ }^{30}$. Suite à ces événements, un «mur de paix » a été construit en 1986. Depuis, les urbanistes du NIHE sont très attentifs à ne pas modifier la géographie confessionnelle. Il s'agit cependant moins de préserver les territoires ethno-territoriaux que d'assurer une bonne gestion du parc et de maximiser le retour sur investissement, en évitant la vacance et les destructions liées au conflit.

Cela se traduit néanmoins par le respect des équilibres de population. Aussi, les autorités veillent à stabiliser la population protestante dans l'inner city de Belfast. A Suffolk, seule enclave protestante de Belfast-ouest, le NIHE s'est attaché à maintenir la population malgré le déséquilibre démographique. Les protestants ont progressivement quitté le quartier depuis le début des Troubles, tandis que le quartier catholique voisin, Leenadon, ne cesse de s'étendre. Un mur construit dans les années 1970 sépare les deux communautés sur l'artère principale de Stewartstown Road. A partir des années 1980, le secteur privé investit dans Black's Road, à proximité immédiate de Suffolk, et

27. Entretien avec le Director of Development NIHE, 20 août 1999.

28 . Jarman N., O'Halloran C., "Recreational Rioting: Young People, Interface Areas and Violence", Child Care in Practice, vol.7, n'11, 2001, p. 2.

29. Entretien avec le Director of HousingPolicy NIHE, 14 juin 1999 ; entretien avec le District Manager, District 6, NIHE, 21 février 2000.

30. Manor Street RDA 42 planning and feasibility study. 
les logements sont acquis par des catholiques. Suffolk est alors une enclave d'habitat social isolée des autres quartiers protestants. Le sentiment des habitants est celui d'une accessibilité limitée du quartier et de l'insécurité.

Au début des années 1980, un processus de réhabilitation du quartier d'habitat social est entamé. Cette démarche est contraire à la rationalité administrative et contrainte par le maintien des territoires confessionnels. Une solution envisageable aurait été le relogement des protestants dans un autre quartier et l'attribution des logements sociaux rénovés à des catholiques. La communauté catholique fait d'ailleurs pression sur les autorités locales pour l'attribution des logements. Les bénéfices d'une telle option sont évidents. La rentabilité financière de l'opération aurait été assurée. La pression sur la liste d'attente catholique de Belfast-ouest aurait été soulagée (14\% des candidats auraient été logés). Le commissariat du quartier aurait vu sa tâche facilitée par la baisse des tensions. La création d'une zone catholique homogène aurait eu de nombreux avantages mais elle est politiquement impossible. Elle aurait soulevé non seulement la résistance des habitants de Suffolk, mais aussi celle de la communauté protestante dans son ensemble et de ses représentants institutionnels ou informels. Le NIHE craint d'encourager l'intimidation et de déclencher une «épuration ethnique » menée par les paramilitaires. Il choisit donc la démarche inverse. Un programme de réhabilitation des logements, des espaces extérieurs et de la voirie est lancé, ainsi que l'implantation de services de proximité. Malgré cela, la baisse de population se poursuit dans les années 1990 31. Les autres projets de rénovation de l'habitat de l'inner city affirment la volonté de maintenir la communauté protestante. Les pouvoirs publics ont choisi d'assurer la stabilité confessionnelle sur le territoire urbain. Ce choix est contraire à la satisfaction des besoins objectifs en logements. De plus, cette attitude a des coûts sociaux très importants, notamment la duplication des services urbains et contribue à la pérennisation des murs.

Avec le processus de paix, les protestants les plus marginalisés socio-économiquement ont le sentiment accru d'être laissés pour compte, au profit des catholiques, dont la montée en puissance politique, sociale et économique est indéniable mais qui ne résorbe pourtant pas encore tout à fait les inégalités structurelles entre les deux communautés. Ces dissymétries de perceptions et de situations donnent une acuité nouvelle au problème territorial. En 2005, les derniers résidents d'un programme immobilier de Belfast-nord (Torrens Estate) entièrement occupé par des protestants et entouré d'un mur, le quittent ${ }^{32}$. Selon les résidents, leur départ est motivé par la peur et l'intimidation des républicains, alors que pour ces derniers, leur départ a été négocié. Les protestants ont inter-

31. Murtagh B., "Housing Renewal in Belfast", The Planner, Journal of the Royal Town Planning Institute, vol.78, $\mathrm{n}^{\circ} 18,1992$, p. 11.

32. Shirlow P., Murtagh B., Belfast, Segregation, Violence and the City, Londres, Pluto Press, 2006, p. 68. 
prété ceci comme une défaite, le rattachant au discours de déclin et d'exode qui caractérise la lecture politique dominante de l'unionisme radical.

\section{Banalisation et pérennisation des peacelines dans le paysage urbain}

Le maintien des territoires confessionnels a pour corollaire la mise à distance des quartiers et, de fait, la préservation des murs. Mais le NIHE n'a pas de stratégie générale pour la gestion des espaces divisés et ne propose que des solutions ad hoc pour la construction des peacelines. Dans le document intitulé Coping with conflict, publié en 1988, le NIHE fait le point sur les questions de sécurité intervenant dans son action. Il analyse que :

"l'héritage des divisions sociales à Belfast a laissé treize flashpoints ou interfaces où des communautés rivales vivent à proximité les unes des autres. Ce sont des lieux où le conflit se produit fréquemment et où le NIHE cherche à maintenir l'habitat tout en restant impartial ${ }^{33}$. »

Le rapport reconnaît que trois agences gouvernementales sont consultées pour évaluer les risques. Il s'agit du Belfast Development Office (BDO), du Northern Ireland Office (NIO) et de ses agents de liaison, les Civil representative officers, officiers de renseignements généraux qui assurent la liaison entre le NIO et les forces de l'ordre.

La construction des peacelines n'est pas décidée par le NIHE puisque la décision relève de la police, mais celles-ci sont situées sur ses terrains ou des parcelles attenantes et il en est de fait le constructeur. Dans ce contexte, les murs sont maintenus par le NIHE et banalisés. Il existe une tendance longue de l'incorporation des dispositifs de séparation et de cloisonnement dans les opérations de réhabilitation urbaine, les coupures se fondant à présent dans le paysage. En effet, depuis le début des années 1980, le NIHE incorpore les dispositifs existants, souvent sommaires, faits de tôles ondulées, de barbelés et de béton, dans ses opérations de réhabilitation urbaine. Les peacelines, intégrées dans le projet, se confondent avec un aménagement ordinaire. A partir de la fin des années 1980, des «barrières environnementales alternatives » consistant en des murs de briques ornés de motifs, grilles et zones plantées sont de véritables compositions paysagères. Les peacelines rénovées et banalisées (murs de briques et de béton agrémentés de plantations constituant des jardins inaccessibles) sont issues du service d'urbanisme et d'architecture de cet organisme, qui a appliqué au cas par cas ce schéma pour la gestion de son parc de logements sociaux. Des considérations foncières et immobilières président aussi aux investissements réalisés pour la construction de murs. En effet, la

33. NIHE, Coping with conflict..., op. cit., p. 2. 
barrière a pour fonction de protéger les biens immobiliers de la dégradation (en prévenant les jets de pierres et de cocktails Molotov) et, en donnant des garanties aux locataires, de les rassurer et de limiter ainsi la vacance des logements situés près des peacelines. La logique immobilière semble confirmée par le fait qu'après 2005, des promoteurs privés ayant acheté les terrains au NIHE construisent des pavillons en accession à la propriété sur des parcelles libres jouxtant les peacelines.

Le coût de construction et de maintenance de ces structures est impossible à évaluer. Des estimations existent, mais elles sont lacunaires. Brendan Murtagh estime que le coût total des treize peacelines construites jusqu'en 1994 s'élève à près de deux millions de livres ${ }^{34}$. Pour une peaceline, les coûts s'élèvent de 16000 livres ${ }^{35}$ à 750000 livres ${ }^{36}$. Michel Foucher cite un montant de 300 euros pour 500 mètres ${ }^{37}$. Non seulement les murs représentent un fardeau supplémentaire dans les budgets publics, mais ils induisent une duplication des services urbains dont le coût est estimé à 1,5 million de livres par an ${ }^{38}$.

Le NIHE utilise en outre la mise à distance des territoires confessionnels, en aménageant des zones comprenant des espaces libres ou un usage autre que l'habitat considéré comme neutre : routes, résidences pour personnes âgées, zones industrielles ou artisanales. Cela est rendu possible par le dépeuplement des zones résidentielles protestantes, les terrains libres permettent une dédensification lors des réhabilitations de quartiers. L'aménagement de zones artisanales ou industrielles sur le modèle des pépinières d'entreprises ou des parcs industriels doit non seulement assurer la revitalisation économique du quartier, mais aussi justifier et permettre l'altération partielle de la géographie confessionnelle.

Pour désigner ces procédés - rénovation, barrières environnementales et zonages alternatifs -, les cadres du NIHE utilisent le terme de softening, dont la traduction littérale est «atténuation ». Ce terme contient l'idée de rendre plus douce et plus floue une séparation, plutôt tranchée visuellement. Il en résulte une banalisation du dispositif. La position du NIHE est que les peacelines sont souhaitées par les communautés car elles leur procurent un sentiment de sécurité. Mais elles ne représentent pas une démarcation irréversible des territoires, bien qu'elles aient des effets matériels et symboliques importants.

34. A partir des chiffres que lui a fournis le DOE, responsable de la planification urbaine. Murtagh B., Ethnic Space and the Challenge to Land Use Planning: A Study of Belfast's Peace Lines, Research paper n7, Centre for Policy Research, University of Ulster, 1994, p. 25.

35. Pour Bryson Street.

36. Pour Springmartin Road. Voir Ballif F., Les Peacelines de Belfast : du maintien de l'ordre à l'aménagement urbain, 1969-2002, thèse soutenue à l'Institut d'urbanisme de Paris, université Paris-XII - Val-de-Marne, 2006, pp. 519-520.

37. Foucher M., op. cit., p. 16.

38. Deloitte, Research into the Financial Cost of the Northern Ireland Divide. 
Les murs contribuent à mettre les populations à distance, autant par la séparation physique que symbolique. En effet, ils ont un effet indéniable de transformation des petites distances à l'échelle de quartiers, en symbole de différentiation politique et culturelle ${ }^{39}$. Ils deviennent aussi «le visage malveillant de ceux qui vivent de l'autre côté 40 ». La rénovation urbaine peut aggraver ou lever les tensions, en cristallisant la division, ou par les débats qu'elle provoque sur l'histoire des lieux. Un phénomène relativement récent, au début des années 2000, dans son caractère systématique et formalisé, est l'implantation de monuments aux morts près des peacelines, même s'il s'appuie sur une longue tradition. La nouveauté est le caractère pérenne de ces monuments, autrefois artisanaux et amovibles ou des fresques peintes sur les pignons des maisons en bandes dans les quartiers ouvriers de la ville. A la pérennité des monuments, répond celle des peacelines celles-ci marquant une tendance à incorporer la souffrance dans la ville, en oblitérant la possibilité de l'oublier. Ils marquent aussi un espace sacré, inamovible et approprié par une communauté.

Pourtant, dans une enquête de janvier 2008 commanditée par l'USIreland Alliance $41,81 \%$ des personnes interrogées (sur un échantillon de 1037 personnes résidant vivant à proximité d'une peaceline), se déclarent en faveur de la destruction du mur : seuls $21 \%$ estiment que cela doit être réalisé immédiatement et $60 \%$ le souhaitent, non pas pour tout de suite, mais quand la situation sera plus «sûre ». $17 \%$ déclarent être indifférents au maintien du mur. La même enquête fait état d'un «fort sentiment que les murs ont aidé les habitants à se sentir plus en sécurité en contribuant à séparer les communautés ». Mais il faut souligner qu'en ne remettant pas en cause les fondements territoriaux du conflit, ces murs contribuent à le perpétuer. Toutefois, avec le processus de paix de 1998, la signification du mur peut évoluer parce que les représentations du conflit ont changé. Avec l'apaisement, la municipalité lance des forums sur la ville partagée (shared city) et promeut les «bonnes relations ( good relations plan) ce qui encourage notamment, couplé avec d'autres programmes, les initiatives locales de développement social et de partage de l'espace urbain. Comme le souligne Trogmorton, un mur sépare, mais peut réunir. Le mur le plus «dur » peut agir comme une «couture » qui réunirait les côtés opposés. "De plus, la signification d'un mur n'est pas un fait objectif, indépendant de toute interprétation ${ }^{42}$. »

39. Shirlow P., Murtagh B., op. cit., p. 57.

40. Feldman A., Formations of Violence, The University of Chicago Press, 1991, p. 37.

41. www.us-irelandalliance.org

42. Trogmorton J., "Where was the Wall then? Where is it now?", Planning Theory and Practice, vol.5, n³, 2004, p. 351 . 